\title{
Listeria Spinal Cord Abscess Responsive to Trimethoprim-Sulfamethoxazole Monotherapy
}

\author{
Fabian H. Rossi, Jennifer White, Ronald Quisling, William J Triggs, \\ Michael S. Okun
}

\begin{abstract}
Objective: To describe an alternative antibiotic regimen for the treatment of central nervous system Listeria monocytogenes infection. Background: Classical treatment of listeria infections of the brain and spinal cord has included ampicillin in combination with gentamicin and chloramphenicol. Antibiotic resistance to L. monocytogenes is extraordinarily low, and the combined risks of nephrotoxicity, ototoxicity, and agranulocytosis in an already critically ill patient make the potential use of trimethoprim-sulfamethoxazole monotherapy for coverage or treatment of listeria an important alternative. Methods: Case report. Results: A 58-year-old woman presented with a two-week history of progressive quadriplegia. Gadolinium enhanced MRI showed diffuse edema of the cervical and thoracic spine with ring-enhancing lesions. Cerebrospinal fluid and blood cultures both grew $L$. monocytogenes. Spinal cord biopsy of the lesion revealed inflammation with necrosis and also grew listeria. Intravenous trimethoprim-sulfamethoxazole $(8 \mathrm{mg} / \mathrm{kg}$ in four divided doses) was administered for six weeks with resultant arrest of neurological symptoms and stabilization of the clinical course. Although the patient was quadraparetic she was able to be discharged to a rehabilitation facility. Conclusions: Trimethoprim-sulfamethoxazole monotherapy may be a potential alternative option for critically ill patients with central nervous system L. monocytogenes infection.
\end{abstract}

RÉSUMÉ: Abcès de la moelle épinière à Listeria sensible au triméthoprim-sulfaméthoxazole en monothérapie. Objectif: De décrire un régime antibiotique alternatif pour le traitement de l'infection du système nerveux central par Listeria monocytogenes. Introduction: Le traitement classique des infections du cerveau et de la moelle épinière par Listeria comportait une combinaison d'ampicilline, de gentamicine et de chloramphénicol. La résistance de Listeria monocytogenes à l'antibiothérapie est très faible et les risques combinés de néphrotoxicité, d'ototoxicité et d'agranulocytose chez un patient en état critique font de la monothérapie par le triméthoprimsulfaméthoxazole pour la couverture ou le traitement de la listeriose une alternative importante. Méthodes: Il s'agit d'une observation clinique. Résultats: Une femme âgée de 58 ans a consulté pour une quadriplégie progressive depuis deux semaines. La RMN rehaussée au gadolinium a montré un oedème diffus du rachis cervical et thoracique accompagné de lésions rehaussantes annulaires. Les cultures du liquide céphalo-rachidien et du sang étaient positives pour le Listeria monocytogenes. La biopsie de la lésion de la moelle épinière a montré de l'inflammation avec nécrose et la culture était également positive pour Listeria monocytogenes. La patiente a été traitée pendant six semaines au moyen de triméthoprim-sulfaméthoxazole intraveineux à raison de $8 \mathrm{mg} / \mathrm{kg}$ administré en quatre doses, avec arrêt des symptômes neurologiques et stabilisation de l'évolution clinique. Bien que la patiente était quadriparétique, elle a pu être transférée dans un centre de réadaptation. Conclusions: La monothérapie par le triméthoprim-sulfaméthoxazole peut être une option de rechange chez le patient atteint d'une infection du système nerveux central par le Listeria monocytogenes dont l'état est critique.

Can. J. Neurol. Sci. 2001; 28: 354-356

Listeria monocytogenes is a gram-positive rod found in soil, wastewater and sewage. It is present as normal flora in the intestinal system of mammals as well as in birds and fish. ${ }^{1}$ Listeriosis is usually contracted by ingesting contaminated food or water. It is then hematogenously disseminated. Meningitis is the most common clinical presentation of listeriosis accounting
From the Department of Neurology (FHR, WJT, MSO), Department of Medicine, Division of Infectious Diseases (JW), Department of Radiology (RQ), University of Florida, Gainesville, Fl; Emory University, Department of Neurology (MSO), Atlanta, GA, USA

ReCEIVED DeCEMBER 13, 2000. ACCEPTED IN FINAL FORM June 20, 2001. Reprint requests to: Michael S. Okun, 1841 Clifton Road NE, Wesley Woods Health Science Center Building. Atlanta, GA 30329 USA 
for half of all clinical features. ${ }^{1}$ Brain and spinal cord abscesses are potential serious complications of listeriosis. ${ }^{2}$ Ampicillin in combination with chloramphenicol or gentamicin has been the gold standard treatment of listeria infection. Nephrotoxicity, ototoxicity, and agranulocytosis may limit the use of this combination of drugs in a critically ill patient. ${ }^{3}$ We report a case of Listeria monocytogenes in the cervical and thoracic spinal cord treated with trimethoprim-sulfamethoxazole monotherapy. It was decided that because antibiotic resistance to $L$. monocytogenes was low and the patient was clinically improving that continuation of monotherapy with trimethoprimsulfamethoxazole would be appropriate treatment for her infection.

\section{CASe Report}

A 58-year-old woman presented with severe neck pain and right arm numbness. She also complained of right hand weakness. Cervical computed tomography revealed C6-7 spinal canal stenosis with bilateral narrowing of the neural foramen. A trial of steroids was given without effect. Her weakness progressed over one week ultimately leading to flaccidity in the upper extremities as well as lower extremity spasticity. She had bilateral extensor plantar responses. Magnetic resonance image (MRI)revealed an area of increased $\mathrm{T} 2$ signal from $\mathrm{C} 1$ to $\mathrm{T} 7$ of the cervical and thoracic spinal cord. (Figure 1A). Lumbar puncture revealed a white blood cell count of 713 , a red blood cell count of 3500 , protein $234 \mathrm{mg} / \mathrm{dl}$ and glucose of $75 \mathrm{mg} / \mathrm{dl}$ (serum 203). Complete blood count, including white blood cell count, was normal. Cervical laminectomy with a microsurgical biopsy of the spinal cord was performed and revealed necrosis and an acute inflammatory reaction. Cerebrospinal fluid, blood and biopsy culture all grew $L$. monocytogenes. A repeat MRI one week later evidenced ringed enhancing lesions in the cervical and thoracic spinal cord (Figure 1B-C). Trimethroprim-sulfamethoxazole intravenously $(8 \mathrm{mg} / \mathrm{kg}$ in four divided doses) was continued as monotherapy since the listeria infection was sensitive to this drug by laboratory testing. Meningeal signs resolved and cerebrospinal fluid cultures became negative six weeks after therapy. The neurological exam after treatment showed a modest improvement in bilateral finger movements. The patient was able to be weaned from the respirator and discharged to a rehabilitation facility after the completion of antibiotic treatment.

\section{DISCUSSION}

Meningitis is the most common clinical presentation of listeria infection, accounting for half of all the clinical symptoms reported. ${ }^{1}$ Five to ten percent of the community-acquired acute bacterial meningitis cases are caused by L. monocytogenes. ${ }^{4}$ Cerebritis, brain abscess and rhomboencephalitis are also common complications of neurolisteriosis. ${ }^{1}$ Brain abscess is a rare manifestation affecting predominantly those who are immunosuppressed or have renal insufficiency, alcoholism or collagen vascular disease. ${ }^{5}$ Spinal cord abscess ${ }^{5-11}$ mainly affects adult males (male:female ratio 6:2) with the cervical and thoracic spine being the most common. Half of all patients with listeria spinal cord abscess are immunocompetent and most have a history of alcoholism or diabetes.

The typical clinical presentation of $L$. monocytogenes spinal

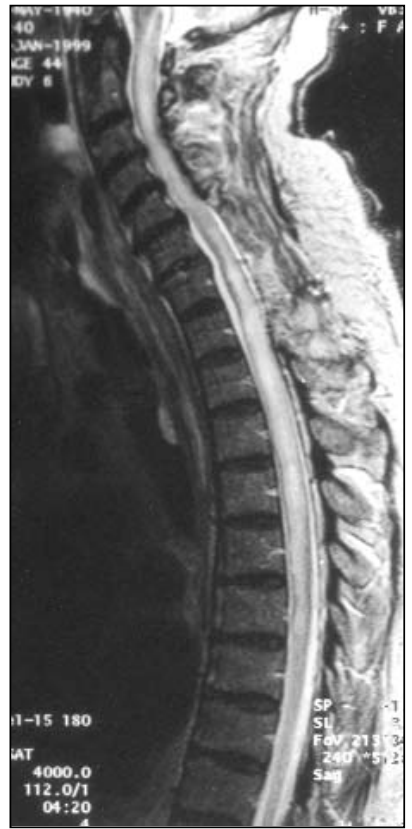

Figure 1A: T2-weighted sagittal MRI image demonstrates diffuse spinal cord edema extending from the cervico-medullary junction to the $T 7$ level of the thoracic spinal cord. There is a mild fusiform enlargement of the spinal cord between $T 1$ and T7. The extra axial spaces appear normal.

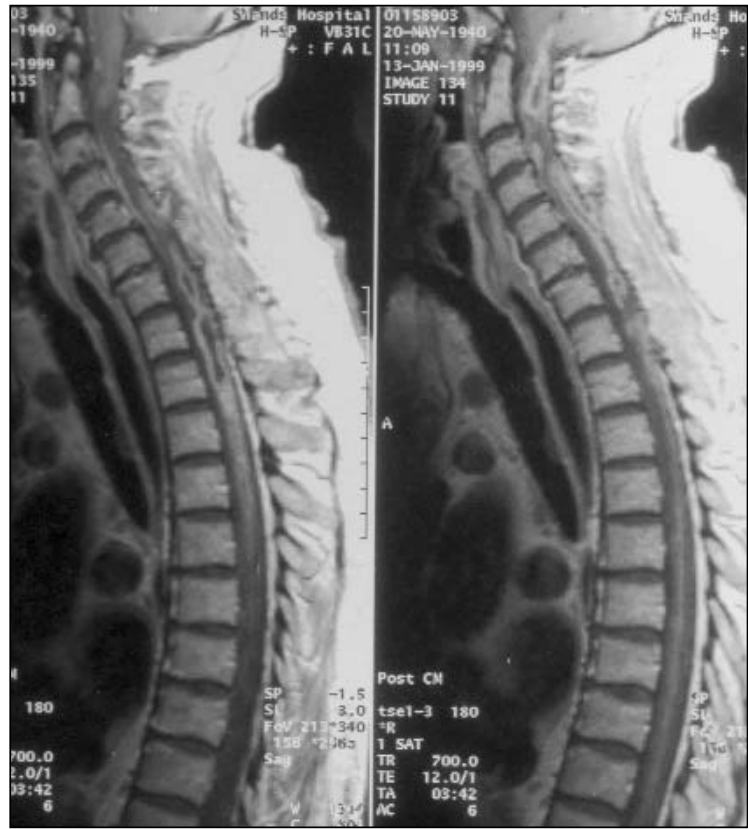

Figure 1B: Gadolinium-enhanced sagittal T1-weighted MRI image demonstrates abnormal contrast enhancement within the spinal cord. There are multiloculated areas of central lucency consistent with a suppurative process. There is evidence of minimal pial enhancement along the anterior surface of the upper cervical cord.

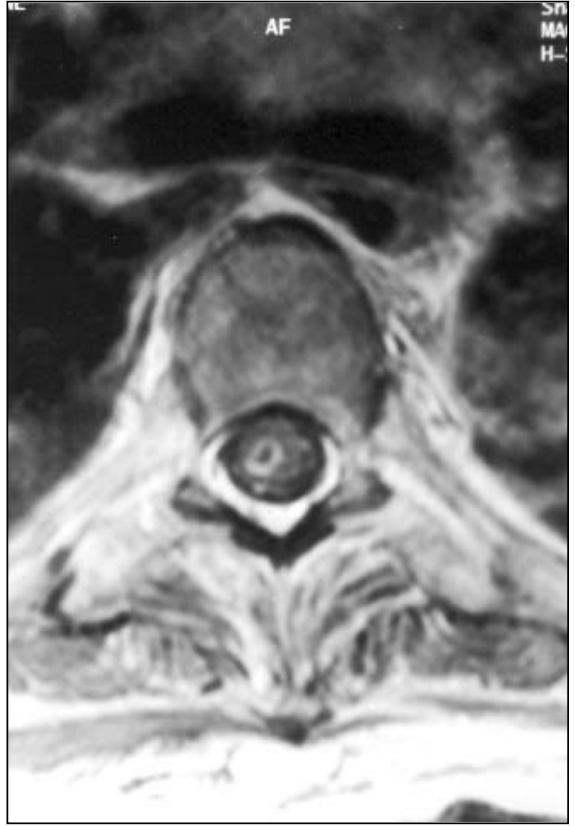

Figure 1C: T1-weighted gadolinium-enhanced axial MRI image obtained at the T6 level confirms the intra-axial location and the crosssectional appearance of a spinal cord abscess. Ring enhancement surrounds central areas of necrosis. 
cord abscess is an acute or subacute myelopathy with meningeal symptoms, malaise, fever, nausea, vomiting and confusion. The organism grows in blood, cerebrospinal fluid or tissue culture. However, it is rarely proven to be present in all three mediums. Cerebrospinal fluid often reveals an elevated protein, lymphocytosis and a normal or reduced glucose. Typical MRI demonstrates ring-enhancing lesion in the spine but may be nondiagnostic, making serial studies crucial in cases where listeria is suspected.

Treatment of listeria spinal cord abscess typically consists of multiple antibiotic combinations. The most common combination is ampicillin combined with gentamicin or chloramphenicol. ${ }^{3}$ These combinations can present difficulties in the management of the critically ill patient, particularly with regard to nephrotoxicity, ototoxicity, and agranulocystosis. Recently the alternative of trimethoprim-sulfamethoxazole in combination with gentamicin was also reported as successful. ${ }^{11}$ Despite usual excellent control of the infection and reversal of the meningitis, permanent neurological sequela is usually present if the spinal cord is involved. It has been suggested that lack of intracellular penetration of antibiotics in the spinal cord, as well as in leptomeningeal and penetrating blood vessels, contributes to poor neurological recovery. ${ }^{9}$

Our case of listeria cervical and thoracic spinal cord abscess was treated successfully with trimethoprim-sulfamethoxazole monotherapy. Because of the sensitivity, safety, and excellent cerebrospinal fluid penetration of this antibiotic we chose monotherapy for this patient. Antibiotic resistance to $L$. monocytogenes has been recently reported as low $(0.6 \%)$ as compared to listeria innocua $(19.5 \%)^{12}$ making the choice of monotherapy after identification of the organism a sensible alternative. Monotherapy with this drug also may be considered as an alternative to combination therapy particularly when treating a critically ill patient with significant comorbidities. Listeria spinal cord abscess should be considered in any case of acute or subacute meningitis complicated with myelopathy or rhombencephalopathy regardless of the immunological status of the patient. Blood and cerebrospinal fluid cultures may be negative, making the diagnosis difficult. Administration of intravenous trimethoprim-sulfamethoxazole monotherapy for antibiotic coverage prior to to culture diagnosis of an organism may be a safe and effective way to cover for the possibility of listeria infection. Further reports and studies of the efficacy of monotherapy with trimethoprim-sulfamethoxazole are needed to substantiate its role in the treatment of central nervous system listeria infections.

\section{REFERENCES}

1. Hoeprich PD, Jordan MC, Ronald AR. Listeriosis. In: Infectious Diseases. Fifth ed. Philadelphia: JB Lippincott Co, 1994.

2. Mielke MEA, Held TK, Connor DH, Chandler FW. Unger M. Listeriosis. In: Pathology of Infectious Diseases. Appleton \& Lange Press, 1997; Vol 1: 621-633.

3. Gorbach S, Bartlett JG, Blacklow NR.. Listeria Monocytogenes. In: Infectious Diseases. Second edition: Philadelphia: W.B. Saunders Co Press 1998.

4. Durand ML, Calderwood SB, Weber DJ, et al. Acute bacterial meningitis in adults : a review of 493 episodes. N Engl J Med 1993; 328: 21-28.

5. Morrison RE, Brown J, Gooding RS. Spinal cord abscess caused by listeria monocytogenes. Arch Neurol 1980;37:243-244.

6. Barber M, Okubadejo OA. Maternal and neonatal listeriosis: report of case and brief review of literature of listeriosis in man. Br Med J 1965; 5464:735-738.

7. Kendall MJ, Clarke SW, Smith TW. Spinal abscess due to listeria monocytogenes in a patient with hepatis cirrhosis. J Pathol 1972;107:9-11.

8. King SJ, Jeffree MA. MRI of an abscess of the cervical spinal cord in a case of listeria meningoencephalitis. Neuroradiology 1993;35(7):495-496.

9. Pfadenhauer K, Rossmanith $\mathrm{T}$. Spinal manifestation of neurolisteriosis. J Neurol 1995; 242 (3);153-156.

10. Chu JY, Montanera W, Willisky RA. Listeria spinal cord abscessclinical and MRI findings. Can J Neurol Sci 1996; 23(3):220223.

11. Duprez TP, Grandin CB, Osseman M, Cosnard G, Sindic CJ. MR Monitoring of a medically treated spinal cord abscess presumptively due to listeria monocytogenes. Eur Neurol 1997; 37(4):249-251.

12. Walsh D, Duffy G, Sheridan JJ, Blair IS, McDowell DA. Antibiotic resistance among listeria, including listeria monocytogenes, in retail foods. J Appl Microbiol 2001; 90(4):517-522. 